\title{
CIDME: Short distances measured with long chirp pulses
}

\section{Journal Article}

Author(s):

Doll, Andrin; Qi, Mian; Godt, Adelheid; Jeschke, Gunnar

Publication date:

2016-12

Permanent link:

https://doi.org/10.3929/ethz-a-010745297

Rights / license:

In Copyright - Non-Commercial Use Permitted

Originally published in:

Journal of Magnetic Resonance 273, https://doi.org/10.1016/j.jmr.2016.10.011

Funding acknowledgement:

157034 - Nanometer-range distance constraints for biomolecular complexes - Measurement, uncertainty estimates, and use in modeling (SNF) 


\title{
CIDME: Short distances measured with long chirp pulses
}

\author{
Andrin Doll ${ }^{\mathrm{a}, \mathrm{c}}$, Mian Qi ${ }^{\mathrm{b}}$, Adelheid Godt ${ }^{\mathrm{b}}$, Gunnar Jeschke $\mathrm{e}^{\mathrm{a}, *}$ \\ ${ }^{a}$ Laboratory of Physical Chemistry, ETH Zurich, Vladimir-Prelog-Weg 2, CH-8093 Zurich, Switzerland \\ ${ }^{b}$ Faculty of Chemistry and Center for Molecular Materials, Bielefeld University, Unversitätsstraße 25, 33615 Bielefeld, Germany. \\ ${ }^{c}$ Current Address: Service de physique de l'état condensé, CEA Saclay, 91191 Gif-sur-Yvette, France
}

\begin{abstract}
Frequency-swept pulses have recently been introduced as pump pulses into double electron-electron resonance (DEER) experiments. A limitation of this approach is that the pump pulses need to be short in comparison to dipolar evolution periods. The "chirp-induced dipolar modulation enhancement" (CIDME) pulse sequence introduced in this work circumvents this limitation by means of longitudinal storage during the application of one single or two consecutive pump pulses. The resulting six-pulse sequence is closely related to the five-pulse "relaxation-induced dipolar modulation enhancement" (RIDME) pulse sequence: While dipolar modulation in RIDME is due to stochastic spin flips during longitudinal storage, modulation in CIDME is due to the pump pulse during longitudinal storage.

Experimentally, CIDME is examined for Gd-Gd and nitroxide-nitroxide distance determination using a high-power Q-band spectrometer. Since longitudinal storage results in a 50\% signal loss, comparisons between DEER using short chirp pump pulses of $64 \mathrm{~ns}$ duration and CIDME using longer pump pulses are in favor of DEER. While the lower sensitivity restrains the applicability of CIDME for routine distance determination on high-power spectrometers, this result is not to be generalized to spectrometers having lower power and to specialized "non-routine" applications or different types of spin labels. In particular, the advantage of prolonged CIDME pump pulses is demonstrated for experiments at large frequency offset between the pumped and observed spins. At a frequency separation of $1 \mathrm{GHz}$, a Gd-Gd modulation depth larger than $10 \%$ is achieved, where broadening due to dipolar pseudo-secular contributions becomes largely suppressed. Moreover, a CIDME experiment at deliberately reduced power underlines the potential of the new technique for spectrometers with lower power, as often encountered at higher microwave frequencies. With longitudinal storage times $T$ below $10 \mu \mathrm{s}$, however, CIDME appears rather susceptible to artifacts. For nitroxide-nitroxide experiments, these currently inhibit a faithful data analysis. To facilitate further developments, the artifacts are characterized experimentally. In addition, effects that are specific to the high spin of $S=7 / 2 \mathrm{Gd}$-centers are examined. Herein, population transfer within the observer spin's multiplet due to the pump pulse as well as excitation of dipolar harmonics are discussed.
\end{abstract}

Keywords: chirp pulses, distance determination, sensitivity enhancement

\section{Introduction}

Using pulsed electron paramagnetic resonance (EPR) spectroscopy, the dipolar interaction between a pair of electron spins can be separated from other interactions. The most promi5 nent pulse sequence within this context is the four-pulse double electron-electron resonance (DEER) experiment [1], which is sometimes also referred to as pulsed electron double resonance (PELDOR). This technique allows to probe distances in the lower nanometer range, which provides structural informa10 tion on spin-labeled biomacromolecules or on systems containing paramagnetic co-factors [2-5].

Recently, frequency-swept pulses generated by fast arbitrary waveform generators (AWG) have been incorporated into DEER experiments. These chirp pulses have been well known 15 for their enhanced excitation bandwidth [6,7], such that initial DEER applications made use of frequency-swept pump pulses

\footnotetext{
${ }^{*}$ Corresponding author. Fax: +41-44 6331448

Email address: gjeschke@ethz.ch (Gunnar Jeschke)
}

to enhance the dipolar modulation, i.e. the DEER modulation depth $\lambda$. In particular, significant improvements in $\lambda$ were demonstrated when pumping the broad EPR spectra of $\mathrm{Cu}$ (II), $\mathrm{Co}(\mathrm{II})$, or $\mathrm{Gd}(\mathrm{III})$ [8-10]. Other than the substitution of the pump pulse by a chirp pulse in the four-pulse DEER sequence, extended pulse sequences have been demonstrated to further enhance sensitivity. Namely pre-polarization by population transfer in high-spin systems [11] as well as the use of multiple pump pulses [12] for prolongation of the phase memory time by dynamical decoupling [13].

An important limitation of a chirp pump pulse substituted into four-pulse DEER is the potential interference of the dipolar evolution. In particular, the chirp pump pulse inverts different spin packets at different times, so that the resulting DEER modulation is a superposition of time-shifted contributions. If the time shift amongst these contributions is comparable to the dipolar oscillation period, the resulting modulation does no longer represent the true dipolar modulation.

The duration of the pump pulse therefore needs to be chosen carefully. To date, there is no general rule or experimental pro- 
cedure yet which allows to determine the optimum pulse duration. Nevertheless, the heuristic rule that the pulse duration should be shorter than a quarter of the principal dipolar evolution period [8] to date appears to be adequate. For distances below $3 \mathrm{~nm}$, pump pulse durations on the order of a few tens of nanoseconds are common. In principle, even a short $64 \mathrm{~ns}$ pulse can exhibit remarkable inversion performance over several $100 \mathrm{MHz}$ when centered around the main frequency of the microwave resonator, as demonstrated using high-power instrumentation at both $X$ band [14] and $Q$ band [15]. In a DEER experiment, however, it is not necessarily an optimum strategy to place a broadband pump pulse at the center of the resonator mode, since this pushes the observation frequency of the DEER 50 spin echo to a position of weak detection efficiency. Unless a dual-mode cavity is utilized [16], one would therefore expect that the limitation on the pump pulse duration constrains DEER sensitivity at short distances, since the inversion efficiency of the short pump pulse is poor.

55 For longer distances and towards the upper distance limit of DEER, longer pump pulses become admissible, since the dipolar evolution period scales with $r^{3}$. In principle, the experimental performance in this regime can be enhanced significantly by prolongation of the pulse duration. In particular, one could

60 prolong the pulses to the asymptotic limit where an adiabatic passage is realized [17]. However, it may not be advisable to suppress the short distances too extensively by too long pulses, since the studied system could exhibit relevant conformations at shorter distances than expected. Accordingly, experimental 65 techniques that are free of interference are of high interest.

One way to overcome the limitation on pulse duration is to use multiple pump pulses for refocusing the frequency-dispersion introduced by the chirp pump pulse [9]. However, DEER with multiple pump pulses entails unwanted dipolar evolution path-

70 ways, which complicates data analysis considerably $[13,12]$.

In this publication, a new pulse sequence is introduced that is free of interference and free of unwanted dipolar evolution pathways of the type as encountered in multi-pulse DEER. The main idea for avoiding interference is to freeze dipolar evolu-

75 tion while applying the chirp pump pulse.

The underlying concept is described in Section 1.1, where it will also become clear why the acronym CIDME of the new pulse sequence is close to the so-called RIDME experiment [18]. Experimentally, CIDME is tested with both Gd-Gd spin 80 pairs and nitroxide-nitroxide spin pairs at Q-band frequencies, which are described in Section 2.

Experimental results and discussions for Gd-Gd spin pairs are presented in Section 3.1, where we point at apparent differences between CIDME and RIDME experiments for introductory pur85 poses. Several complications arise from the $S=7 / 2$ high spin state of Gd-based tags. First, pump pulses in CIDME do not only flip the coupled partner spin, but also cause magnetization transfer within the high-spin multiplet of the observer spin (Section 3.1.1). Second, long and intense CIDME pump pulses 9o may excite higher harmonics of the involved dipolar frequencies (Section 3.1.2). After taking this into account, the sensitivity of Gd-Gd CIDME is compared to Gd-Gd DEER in Section 3.1.3 and an application to short distances is presented in Sec- tion 3.1.4.

Results and discussion for nitroxide-nitroxide pairs are presented in Section 3.2, where the sensitivity of CIDME is compared to optimized DEER schemes. Overall conclusions to the presented experiments as well as potential future applications for CIDME are given in Section 4. Complementary details and discussions on this manuscript are presented in the supporting information (SI) and there are references to the contents of the SI throughout this manuscript.

\subsection{The CIDME pulse sequence}

Coherent evolution can be frozen by means of longitudinal storage, which is the basis of Mims ENDOR [19], RIDME [18], and field-step DEER [20]. In particular, one can introduce a $\left(\frac{\pi}{2}\right)-T-\left(\frac{\pi}{2}\right)$ block into the four-pulse DEER sequence, as shown in the top row of Fig. 1. The first $\frac{\pi}{2}$ pulse at (3) projects and translates electron coherence to electron population, while the second $\frac{\pi}{2}$ pulse at (4) restores the projected electron coherence for generation of the relevant echo that is marked with an arrow (see also below in Section 3.1.1). During the storage time $T$, there is therefore no contribution from coherent evolution of the electron spin to the echo of interest. If one single or two consecutive pump pulses are applied during the storage time $T$, the reversal of coherent dipolar evolution takes place at the unique time instant (4) when coherence is restored. The consecutive chirp pulses shown in the bottom row of Fig. 1 do therefore not cause interference and can have pulse durations beyond the dipolar evolution period.

Notably, the sequence described above without any pump pulse corresponds to the dead-time free RIDME sequence [21], where the acronym RIDME stands for relaxation-induced dipolar modulation enhancement [18]. In RIDME, longitudinal relaxation events during the storage time $T$ flip the coupled spin and reverse the dipolar evolution of the observed spin at (4). The storage time $T$ should therefore be on the order of the longitudinal relaxation time of the unobserved $B$ spins. Accordingly, the experimental conditions for RIDME, most critically the temperature, need to be optimized with respect to the properties of the

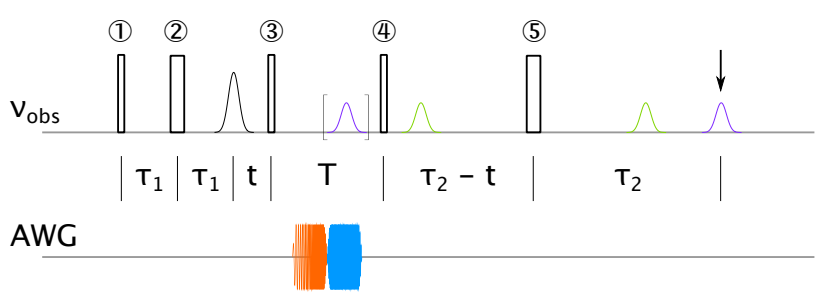

Figure 1: The CIDME pulse sequence, showing the five observer pulses and the pump pulse during the $T$ interval, which are here two consecutive chirp pulses for pumping Gd-based tags. The duration of the observer pulses is illustrated according to their flip angles of either $\frac{\pi}{2}$ or $\pi$. The positions of important echoes are indicated by the Gaussian curves. The detected echo is a refocused virtual echo (violet) and marked by the black vertical arrow. The refocused stimulated echo (green) is removed by the phase cycle described in [21]. The delay $T$ in CIDME is determined by the duration of the pump pulses plus twice an additional pulse gap given by $\tau_{\text {gap }}$ (see also sequence implementation below in Section 2.2). 
spin pair under investigation. A major difference of RIDME as compared to DEER is that the modulation depth does no longer depend on the bandwidth of the pump pulse, which is favorable for distance determination involving transition metal and lanthanide ions with broad spectra. A number of recent examples are summarized in [22]. Due to the similarity to RIDME, the new pulse sequence with the AWG pulses is termed chirpinduced dipolar modulation enhancement, which is abbreviated as CIDME.

Another experiment that is closely related to the CIDME pulse sequence is the field-step DEER/PELDOR experiment introduced in [20]. In this experiment, the pump pulse is applied at a different field during longitudinal storage in a stimulated echo sequence. Notably, also a field-swept pump pulse for enhanced modulation depth has been demonstrated. In this respect, CIDME is the frequency-swept equivalent of this earlier field-swept approach, additionally with an observer sequence that is free of dead-time restrictions and with the option of consecutive pump pulses.

\section{Materials and methods}

\subsection{Sample preparation}

The synthesis of the Gd-rulers $\mathbf{1}_{\mathbf{1}}$ and $\mathbf{1}_{\mathbf{3}}$ has been detailed elsewhere [23]. In these compounds, the $\mathrm{Gd}$ ions are coordinated with the ligand PyMTA. The chemical structure is llustrated in Fig. 2. The stock solutions of Gd-ruler $\mathbf{1}_{\mathbf{1}}$ and $\mathbf{1}_{3}$ had concentrations of $5 \mathrm{mM}$ in $\mathrm{D}_{2} \mathrm{O}(\mathrm{pH}$ 8.0). For the EPR experiments, the stock solutions were diluted with $\mathrm{D}_{2} \mathrm{O}$ and glycerol- $\mathrm{d}_{8}(1: 1$ in volume) to a concentration of $50 \mu \mathrm{M}$ and 40 $\mu \mathrm{l}$ of the solution were filled into $3 \mathrm{~mm}$ outer-diameter tubes. Nitroxide-nitroxide experiments utilized a spin-labeled WALP23 polypeptide (Pepceuticals Ltd., Leicester, UK) inserted into DOPC bilayers. A detailed description of the preparation of this compound will be given elsewhere. In brief, spin labeling of WALP23 with MTSL at residues 7 and 22 was 165 performed according to the previous description in [24]. The concentration to $50 \mu \mathrm{M}$ in a protonated buffer was prepared as described in [25].

\subsection{Dipolar spectroscopy at $Q$ band}

Experiments were performed on a power-upgraded Bruker 170 Elexsys spectrometer [26] with an extension by a $12 \mathrm{GSa} / \mathrm{s}$ AWG [11]. Experiments with Gd-rulers were performed at 10 $\mathrm{K}$ using a repetition rate of $2.9 \mathrm{kHz}$, while experiments with nitroxide-labeled polypeptide were performed at $50 \mathrm{~K}$ at a repetition rate of $280 \mathrm{~Hz}$.

175 For DEER reference experiments, the four-pulse DEER sequence $(\pi / 2)_{\mathrm{obs}}-\tau_{1}-(\pi)_{\mathrm{obs}}-\left(\tau_{1}+t\right)-(\pi)_{\text {pump }}-\left(\tau_{2}-t\right)-$ $(\pi)_{\mathrm{obs}}-\tau_{2}-$ echo was used [1]. Unless explicitly indicated otherwise, we used $t_{\mathrm{p}}=12 \mathrm{~ns}$ for all observation pulses, $\tau_{1}=400$ $\mathrm{ns}, \mathrm{a}[+(+x),-(-x)]$ phase cycle on the first pulse, and an increment in the dipolar evolution time $t$ by $\Delta t=8 \mathrm{~ns}$.

The CIDME sequence illustrated in Fig. 1 is parametrized as $(\pi / 2)_{\mathrm{obs}}-\tau_{1}-(\pi)_{\mathrm{obs}}-\left(\tau_{1}+t\right)-(\pi / 2)_{\mathrm{obs}}-\tau_{\text {gap }}-(\pi)_{\text {pump }}-\tau_{\text {gap }}-$ $(\pi / 2)_{\mathrm{obs}}-\left(\tau_{2}-t\right)-(\pi)_{\mathrm{obs}}-\tau_{2}-$ echo and was implemented

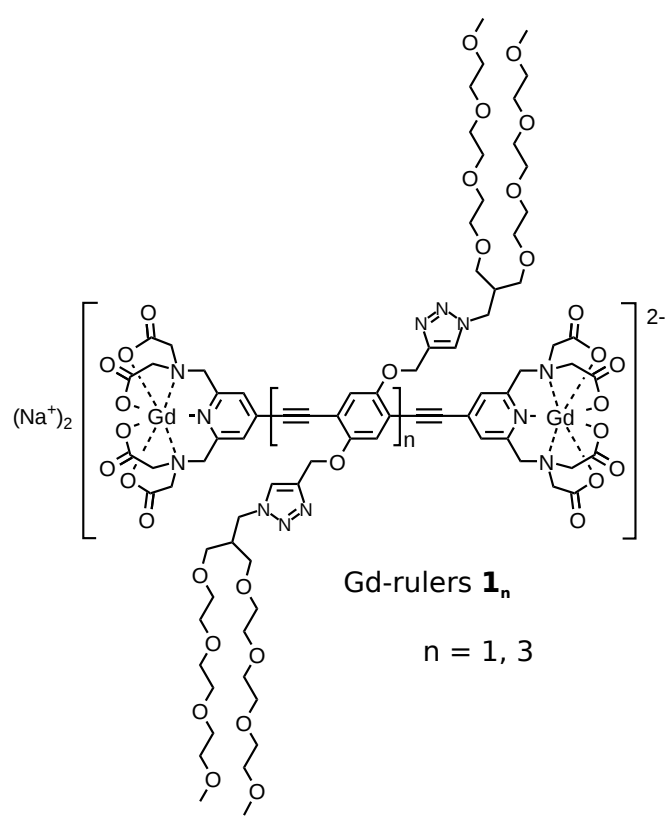

Figure 2: General structures of Gd-rulers $\mathbf{1}_{\mathbf{n}}$, where Gd-ruler $\mathbf{1}_{\mathbf{1}}$ and $\mathbf{1}_{3}$ were used in the EPR experiments within this study. The branched oligo(ethyleneglycol) side chains facilitate solubility in water/glycerol. The poly(para-phenyleneethynylene) unit is repeated $\mathbf{n}$ times, which results in most probable distances of $2.1 \mathrm{~nm}$ and $3.4 \mathrm{~nm}$ for Gd-rulers $\mathbf{1}_{\mathbf{1}}$ and $\mathbf{1}_{\mathbf{3}}$, respectively.

using the eight-step phase cycle to isolate the refocused virtual echo, as introduced previously [21]. The $\pi / 2$ and $\pi$ observation pulses had durations of $12 \mathrm{~ns}$ and $24 \mathrm{~ns}$, respectively, and originated from the so-called stripline channels in the Bruker Elexsys spectrometer to avoid manual channel calibration. The delay $\tau_{\text {gap }}$ was set to $50 \mathrm{~ns}$ and $250 \mathrm{~ns}$ for Gd-Gd and nitroxidenitroxide CIDME, respectively. For nuclear modulation averaging in Gd-Gd CIDME, the delays $\tau_{1}$ and $\tau_{2}$ were incremented eight times by $28 \mathrm{~ns}$. At one instance, it was of interest to perform the experiment with longer $\pi / 2$ pulses of $48 \mathrm{~ns}$ duration at (3) and (4). An attenuator was therefore temporarily incorporated into the Bruker spectrometer to reduce the amplitude of these stripline channels, while having stronger amplitude on the other channels that were here used for the $12 \mathrm{~ns} \pi / 2$ pulses.

For Gd-Gd CIDME using Gd-ruler $\mathbf{1}_{3}$ and related DEER experiments, we have set $\tau_{1}=400 \mathrm{~ns}$, while $\tau_{2}$ had values between 4.5 and $6 \mu \mathrm{s}$. For Gd-ruler $\mathbf{1}_{1}$, the timing was set $\tau_{1}=400$ ns and $\tau_{2}=4.5 \mu \mathrm{s}$, where $\tau_{2}$ was here 2.25 times longer as required for the acquired dipolar evolution window. This was to avoid a pronounced artifact at $t=0$, which was observed at the minimum required $\tau_{2}=2.0 \mu \mathrm{s}$ (see SI). In the nitroxide-nitroxide CIDME and DEER experiments, the timing was $\tau_{1}=300 \mathrm{~ns}$ and $\tau_{2}=1.5 \mu \mathrm{s}$.

In all pulse sequences, the incoherent pump pulses originated from the AWG, independent on whether monochromatic or chirp pump pulses were used. The nomenclature for referring to pulse parameters throughout this work is as described previously (see for instance Section 2.2.2 in [10]). Accordingly, chirp pulses with upward sweep direction sweep from an initial frequency $f_{1}$ to a final frequency $f_{2}$, which corresponds to a 
sweep range $\Delta f=f_{2}-f_{1}$, where all frequencies are indicated to observation frequency. Chirp pulses with downward sweep direction sweep from $f_{2}$ to $f_{1}$.

Gd-Gd distance determination was performed at a resonator bandwidth $(3 \mathrm{~dB})$ on the order of $250 \mathrm{MHz}$. The chirp pulses in this context had sweep widths $\Delta f$ larger than the function of the resonator, as detailed elsewhere $[8,10]$. This bandwidth-compensation establishes an offset-independent adiabaticity factor and facilitates a theoretical estimate of the flipangle of the chirp pulse [17]. During the initial and final

$225 t_{\text {rise }}=10 \mathrm{~ns}$ of the pulse, the amplitude was apodized by a quarter period of a sine function [27]. The static field was set such that the observation position coincided with the maximum of the spectrum [28].

For nitroxide-nitroxide distance determination, we made use

230 of hyperbolic secant pulses (HS) to minimize residual spectral overlap between the excitation profiles of pump and observation pulses. Since residual spectral overlap is only critical at pump frequencies close to the observation position, we used a new type of HS pulse, namely an asymmetric HS pulse. This 235 pulse combines a HS pulse of a higher order [29] at the most distant pump frequencies with a conventional HS pulse [30, 6] of order 1 at the closest pump frequencies. In the case here, the pulse was a HS pulse of order 6 during the first half of the pulse, and a conventional HS pulse of order 1 during the second half of the pulse. Throughout this work, we will refer to this kind of pulse as an asymmetric $\operatorname{HS}\{6,1\}$ pump pulse. The amplitude and frequency modulation functions of this pulse are illustrated in the SI and detailed account on these pulses will be given elsewhere. Notice that for this $\operatorname{HS}\{6,1\}$ pulse, we did not compensate for the resonator with a bandwidth on the order of $100 \mathrm{MHz}$. The positioning of the pulses with respect to the nitroxide spectrum is detailed below in Fig. 8a.

\section{Results and Discussion}

\section{1. $G d-G d C I D M E$}

The performance of Gd-Gd CIDME was tested at Q band with solutions $(50 \mu \mathrm{M})$ of Gd-rulers $\mathbf{1}_{\mathbf{1}}$ and $\mathbf{1}_{\mathbf{3}}$. These compounds consist of two Gd-PyMTA complexes which are kept at a distance of $2.1 \mathrm{~nm}$ and $3.4 \mathrm{~nm}$, respectively, through a rather stiff spacer (see Fig. 2). RIDME experiments at W band with this type of ruler have been reported previously [31]. The apparent differences and similarities between RIDME and CIDME are illustrated in Fig. 3a. The black curve corresponds to a CIDME experiment performed with one single chirp of $128 \mathrm{~ns}$ duration, whereas the gray curve corresponds to a RIDME experiment performed with $T=10.128 \mu \mathrm{s}$. Both curves feature dipolar and nuclear modulations: The fast modulation corresponds to deuterium nuclei. The slow modulation is due to the $3.4 \mathrm{~nm}$ distance between the two Gd complexes.

An apparent difference between the two curves is the steeper background decay in the RIDME data. This decay is due to relaxation processes during the long $T$ interval on the order of 10 $\mu$ s for the RIDME data. In general, an important decay mechanism during $T$ is spectral diffusion due to coupled nuclei [21].
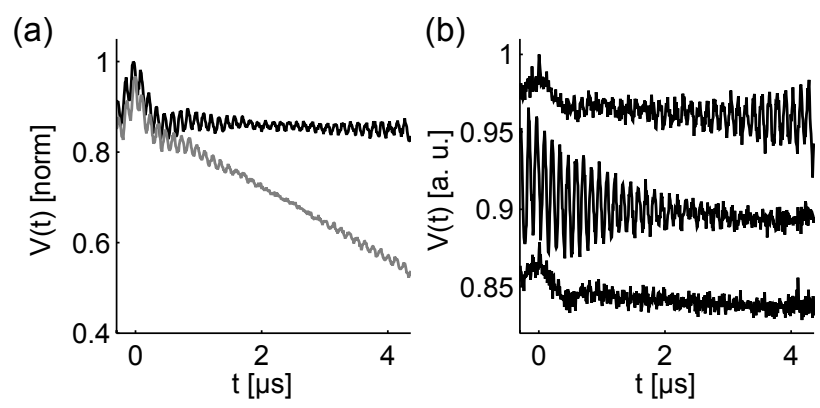

Figure 3: Basic features of the CIDME experiment with Gd-ruler $\mathbf{1}_{\mathbf{3}}$ in deuterated buffer solution. (a) CIDME (black) and RIDME (gray) at Q band. The delay $T$ was $228 \mathrm{~ns}$ and $10.128 \mu$ s for CIDME and RIDME, respectively. The pump pulse for CIDME was parameterized by $\Delta f=800 \mathrm{MHz}, f_{2}=-250$ $\mathrm{MHz}, t_{\mathrm{p}}=128 \mathrm{~ns}$ and $t_{\text {rise }}=10 \mathrm{~ns}$. (b) Removal of nuclear modulations in RIDME/CIDME by summing up eight datasets with increment in $\tau_{1}$ (top), with increment in $\tau_{2}$ (middle), and with increment in both $\tau_{1}$ and $\tau_{2}$ (bottom). The increments for modulation averaging were $28 \mathrm{~ns}$. Here, there was no pump pulse during the delay $T=228 \mathrm{~ns}$.

For the case of Gd-Gd RIDME, an accurate model for this decay is part of ongoing research [31]. Since CIDME has much shorter $T$ intervals, such contributions to the background decay are less important and are not considered in detail within this context. Moreover, a comparison of sensitivity between CIDME and RIDME for Gd-based tags is not within the scope of this work. In particular, the experimental conditions for the data in Fig. 3a were chosen such as to provide comparable modulation depths for introductory purposes.

Nuclear modulations in RIDME are removed by either changing to higher fields, where modulations are diminished, or by division of two measurements performed under different conditions $[21,22]$. Here, the nuclear modulations are suppressed by summing up data with different delays $\tau_{1}$ and $\tau_{2}$, as suggested recently [32]. RIDME traces $(T=228 \mathrm{~ns})$ with different approaches for nuclear modulation averaging are shown in Fig. 3b. The top trace was obtained by averaging over $\tau_{1}$, which cancels nuclear modulations that evolve between (1) and (3). However, the obtained data still exhibit pronounced nuclear modulation towards the end of the curve. These modulations are due to evolution between the virtual echo and the refocused virtual echo and can be removed by averaging over $\tau_{2}$ (middle). Simultaneous averaging of both $\tau_{1}$ and $\tau_{2}$ (bottom) therefore allows for rather good suppression of nuclear modulation artifacts.

With nuclear modulation removed, a residual dipolar modulation of a few percent remains in the data. In a CIDME experiment, where time intervals $T$ can range up to few microseconds, a small fraction of the dipolar modulation therefore stems from this residual contribution. Partially, this residual contribution is due to stochastic spin flips during the short $T$ interval - in other words due to RIDME. However, the $\pi / 2$ pulses of the $\pi / 2-T-\pi / 2$ block in the RIDME pulse sequence can also act as pump pulses $[18,33,20]$, which is relevant under our experimental conditions (see SI). The residual dipolar modulation for RIDME at short $T$ is therefore not only due to stochastic spin flips, but also due to the excitation profiles of the in- 
volved pulses. Besides this residual dipolar modulation, the modulation-averaged trace also reveals artifacts at $t=0$ as well as at $t=\tau_{2}-\tau_{1}=4.1 \mu \mathrm{s}$. For the case discussed here, these are presumably due to improper cancellation of unwanted echoes due to non-ideal phase cycling.

\subsubsection{Echo reduction and pump-observer separation}

While echo decay mechanisms driven by relaxation during long intervals $T$ are less important for CIDME than for RIDME, loss mechanisms induced by the pump pulse need to be considered. For Gd-Gd DEER with a chirp pump pulse, coherence transfer due to inversion of the observed $A$ spin's neighbor transitions was found to be an important reduction mechanism [10]. The underlying coherence transfer mechanism is the same as proposed previously for Gd-nitroxide DEER with monochromatic pulses [34]. For CIDME, a similar effect driven by population transfer is anticipated. For ease of discussion, the presentation of experimental data precedes the details on this population transfer mechanism.

For a direct comparison of the echo reduction effects in DEER and CIDME, the dependence of the echo intensity on the offset between pump and observer was recorded. In accordance with [10], $V_{0}$ is the echo amplitude in the presence of a pump pulse at $t=0$. The echo amplitude in absence of the pump pulse is referred to as $V_{\text {ref. }}$. The normalized echo magnitude $\left|V_{0} / V_{\text {ref }}\right|$ therefore quantifies the net echo reduction effect introduced by the pump pulse.

The experiments were performed using a 128 ns long chirp pump pulse with $\Delta f=750 \mathrm{MHz}$. A pictorial outline of the experiment is shown in Fig. 4a. The solid black curve is the Gd spectrum cast to a frequency axis, which is observed at the central peak by the observer sequence. The orange arrows indicate four representative frequency offsets $f_{2}$ between the pump and observer, which are sampled during the experiment.

The resulting curves are shown in Fig. 4b. The gray curve is the DEER echo reduction and resembles the data shown previously in Fig. 5c in [10]. In particular, there is a gradual loss of echo intensity $V_{0}$ when the pump pulse approaches the observed spins. Due to the sweep width $\Delta f=750 \mathrm{MHz}$ of the chirp pulse, echo reduction for offsets $f_{2}<-100 \mathrm{MHz}$ is primarily due to the aforementioned coherence transfer. In this region, both the inversion efficiency of the pump pulse, which decreases with increasing frequency separation $\left|f_{2}\right|$, as well as the spectral distribution of the relevant neighbor transitions determine the net magnetization transfer (see also [10, 11]). If the pump pulse has spectral overlap with the observed spins, the echo is entirely lost.

Different results are obtained with the CIDME sequence, which are illustrated by the black curve in Fig. 4b. First, a gradual loss in echo intensity is also observed, but it is less pronounced than with DEER. Second, spectral overlap with the observed spins does not completely annihilate the echo. In fact, the pump pulse inverts the observed $A$ spins, which changes the sign of the relevant refocused virtual echo. The observed dependence of the echo magnitude $\left|V_{0} / V_{\text {ref }}\right|$ on pump offset when the pulses have spectral overlap at offsets $f_{2}>0$ is therefore due to a zero crossing while the refocused virtual echo becomes inverted. In Fig.
S1a of the SI, the zero crossing and echo inversion is directly seen in the real part of $V_{0} / V_{\text {ref }}$.

Note that the CIDME experiments showed a dependence of the echo reduction on the sweep direction of the pump pulse. In particular, additional wiggles in $\left|V_{0} / V_{\text {ref }}\right|$ have been observed if an up-chirp was applied (data not shown). All pump pulses in Gd-Gd CIDME therefore had an inverted sweep direction with respect to the upward sweep direction used in DEER, unless explicitly indicated otherwise. Hence, each CIDME pump pulse was swept away from the central transition (CT), while each DEER pump pulse was swept towards the CT.

Another important aspect that is not visible in the plot is intensity of the echo $V_{\text {ref }}$ in absence of the pump pulse, which was here reduced by a factor of 2.6 when changing from DEER to CIDME.

In order to understand the experimental observations for the CIDME sequence, one needs to elaborate further on the spin dynamics of the $\left(\frac{\pi}{2}\right)-T-\left(\frac{\pi}{2}\right)$ block. For a dipolar evolution time $t>0$, the projection and translation of phase-diverging coherence at (3) results in an oscillatory polarization pattern, which is

(a)
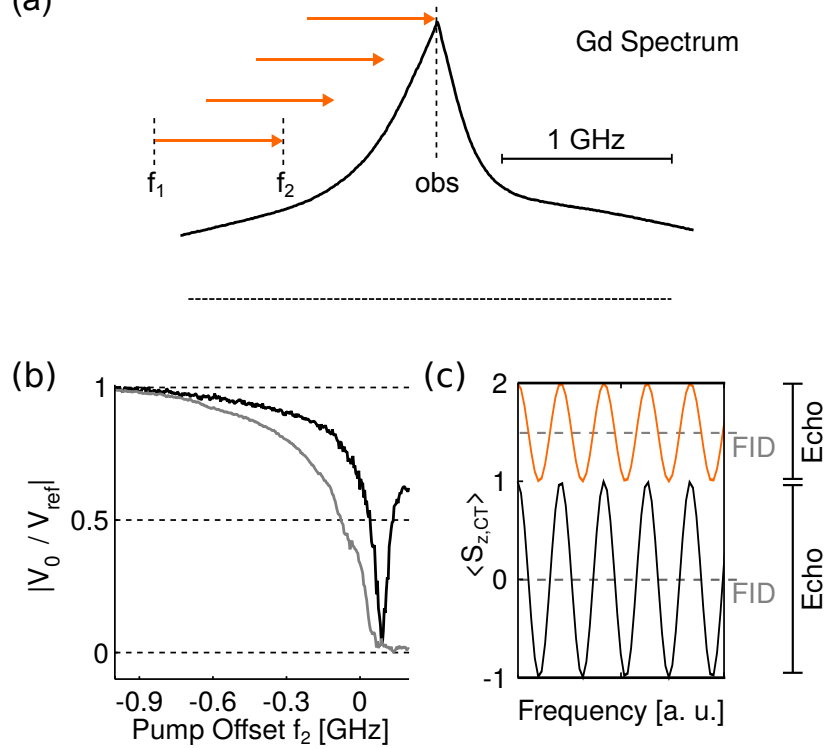

Figure 4: Echo reduction in CIDME experiments using Gd-ruler $\mathbf{1}_{\mathbf{3}}$. (a) Fieldswept echo-detected spectrum cast to a frequency axis (solid black), where the horizontal dashed line corresponds to zero echo intensity. The three vertical dashed lines denote the chirp frequencies $f_{1}, f_{2}$ as well as the observation frequency. The four orange arrows depict the positioning of chirp pulses with $\Delta f$ $=750 \mathrm{MHz}$ at an offset $f_{2}$ of $[-900,-600,-300,0] \mathrm{MHz}$, respectively. (b) Dependence of $\left|V_{0} / V_{\text {ref }}\right|$ on frequency offset for a $128 \mathrm{~ns}$ long chirp with $\Delta f=$ $750 \mathrm{MHz}$ for the CIDME sequence (black) and for the DEER sequence (gray). The observer pulses had durations of $12 \mathrm{~ns}$ and $24 \mathrm{~ns}$ and the experiment was performed according to panel (a) and the description in [10]. Real and imaginary components are shown in Fig. S1a in the SI. The dashed horizontal lines are grid lines (c) Polarization grating on the CT due to projection of coherence by the $\frac{\pi}{2}$ pulse at (3) before (black) and after (orange) inversion of one neighbor transition by the pump pulse. The black vertical rulers on the right indicate the oscillation amplitude of the grating, which results in a phase-converging stimulated echo and a phase-diverging virtual echo once the grating is projected back by the $\frac{\pi}{2}$ pulse at (4). The dashed horizontal lines denote the amplitude of a FID released at (4). See SI for further details on population transfer of a polarization grating. 
referred to as a polarization grating [35]. For phase-diverging coherence on the CT, the translated pattern is illustrated by the black curve in Fig. 4c. The oscillation frequency of the grating is determined by the delay to the preceding refocusing position, which is here the time $t$. The grating encodes the projection of transverse magnetization along one axis. The projection does therefore not preserve the sense of rotation in the transverse plane (with respect to a coordinate system that rotates with the observation frequency). This means that as soon as transverse magnetization is projected to an axis, the sign information of the transverse magnetization's coherence order is lost. Accordingly, it is not possible to discriminate whether the grating originates from phase-diverging or phase-converging coherence. ${ }^{1}$ glected, the grating remains unchanged and coherence is restored at (4). Since the grating cannot carry any information on the sign of the coherence order, this generates both a phaseconverging stimulated echo as well as a phase-diverging virtual echo. The amplitudes and (hypothetical) positions of these echoes are indicated in green and violet around (4) in Fig. 1. Only one half of the initial coherence is therefore detected in the CIDME experiment, which is the main reason for the observed difference by a factor of 2.6 between DEER and CIDME echoes $V_{\text {ref. }}$.

Echo reduction in CIDME is due to population transfer acting on the polarization grating. If the pump pulse inverts only one of the neighbor transitions, one finds the up-shifted grating with half of the oscillation amplitude illustrated in orange in Fig. 4c (see SI for derivation). Unlike in DEER, where inversion of one neighbor transition eliminates the echo, the echo in CIDME is only reduced by a factor of two. With a single pump pulse, CIDME has therefore half of the echo reduction than DEER. The experimental result in Fig. $4 \mathrm{~b}$ is in rather good agreement this for offsets $f_{2}$ below $-100 \mathrm{MHz}$. The dependence of the echo reduction on sweep direction in CIDME is probably also related to population transfer effects.

With pump pulses of smaller sweep width $\Delta f$, DEER echo reduction entails an additional loss mechanism that could be exlained by spatial inhomogeneity of the Bloch-Siegert phase shift [10]. In CIDME, there is no Bloch-Siegert shift due to the pump pulse, both in theory and in experiment. As a consequence, the same type of echo reduction experiment performed with narrower sweep widths $\Delta f$ of $500 \mathrm{MHz}$ and $300 \mathrm{MHz}$ ed less than half of the echo reduction for CIDME than for DEER in those regions where the additional loss mechanism for DEER was expected. These results are discussed in Section 1 of the SI.

A different result is obtained if two consecutive pump pulses ${ }_{430}$ are used. In the limiting case of perfect population inversion of both neighbor transitions, one finds that the polarization grating on the CT is lost. In fact, inversion of both neighbor transitions results in $\left\langle\hat{S}_{z, \mathrm{CT}}\right\rangle=3$, which is due to the population difference between the $+3 / 2$ and the $-3 / 2$ energy level at thermal

\footnotetext{
${ }^{1}$ For the pulse sequence considered here, projection of both phase-diverging and phase-converging spin packets is relevant. In particular, the coherence projected by pulse (3) is phase-diverging for $t>0$ and phase-converging for $t<0$.
}

equilibrium. Within this limiting case of perfect population inversion of both neighbor transitions, echo reduction in DEER and CIDME is the same. However, one has usually a reduced inversion efficiency on each of the neighbor transitions in an experiment with consecutive pulses. When measuring the echo reduction $\left|V_{0} / V_{\text {ref }}\right|$ with consecutive pulses, CIDME still had less echo reduction than DEER, but the difference was smaller than a factor of two (see also SI).

Overall, these experimental results corroborate our previous analysis of echo reduction driven by high-spin effects and by an additional mechanism related to Bloch-Siegert phase shifts in [10].

\subsubsection{Modulation depth and dipolar harmonics}

Since CIDME allows for longer pulses and for overlap between pump and excitation frequencies, modulation depth in CIDME can be rather large. The achievable modulation depth as well as an eventual control of higher harmonics of the dipolar frequency are of interest under these conditions.

For one such example, time-domain CIDME data and the regularized distance distribution are shown in blue in Fig. 5. The pump pulse had a duration of 512 ns and covered a range of $\Delta f=1.9 \mathrm{GHz}$. Because this pulse was centered at the observation frequency, an adiabaticity $Q_{\mathrm{CT}}$ on the order of 4.3 has been achieved for the CT. For such a large frequency range, one would expect that a significant number of spins in the states $m_{S}= \pm 3 / 2$ are flipped three times by the pump pulse and undergo a total change of $\Delta m_{S}=3$. The resulting third harmonic of the dipolar frequency will lead to a contribution in distance domain at around $2.35 \mathrm{~nm}$. There is some hint of such a contribution in the distance distribution extracted from this experiment (see the blue curve in panel $b$ at the arrow marked $3^{\text {rd }}$ ). Another set of experiments (orange and green) was performed with a $1 \mu$ s long up-chirp with $\Delta f=1 \mathrm{GHz}$ and $f_{2}=-250 \mathrm{MHz}$, which achieved an adiabaticity $Q_{\mathrm{CT}}$ on the order of 7.7. The experiment shown in green was performed by observation of the
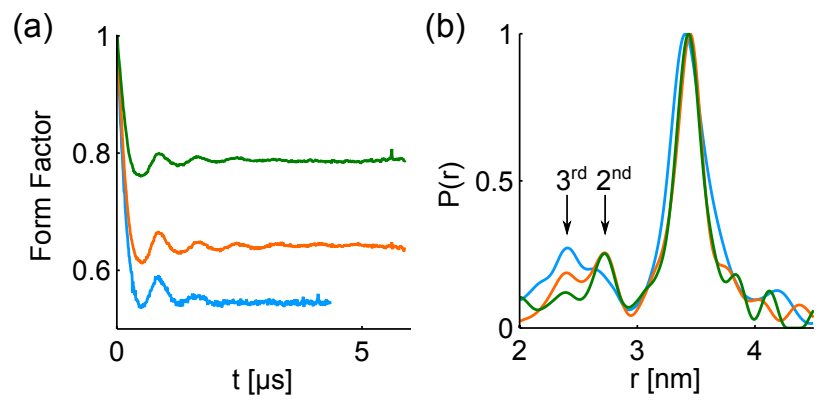

Figure 5: CIDME data of Gd-ruler $\mathbf{1}_{3}$ obtained with long pump pulses for large modulation depths. The pulse parameters were $t_{\mathrm{p}}=512 \mathrm{~ns}$ and $\Delta f=1.9 \mathrm{GHz}$ centered with respect to the observation frequency (blue) and $t_{\mathrm{p}}=1 \mu \mathrm{s}, \Delta f=$ $1 \mathrm{GHz}$ and $f_{2}=-250 \mathrm{MHz}$ (orange and green). Here, all pulses swept the frequency upwards. For the green dataset, the static field $B_{0}$ was reduced by $16 \mathrm{mT}$. The resulting signal loss was compensated by a longer acquisition time and by also using the pump pulse for pre-polarization. (a) Background corrected form factor (b) Regularized distance distribution using a regularization parameter of 1 . The arrows indicate the positions of the $2^{\text {nd }}$ and $3^{\text {rd }}$ harmonic of the principal dipolar frequency translated into distance domain. 
peak of the Gd spectrum, which represents the usual $B_{0}$ setting in these experiments. For the experiment shown in orange, the static field $B_{0}$ was reduced by $16 \mathrm{mT}$ in order to pump the central peak of the Gd spectrum. This corresponds to the field position, where population transfer experiments yielded . [11]). The larger modulation depth of the orange curve as compared to the green curve is therefore due to pumping of the central peak of the Gd spectrum. It should be noted that this is not an optimum approach for a CIDME experiment. In fact, data shown in orange was acquired 20 times longer than the data shown in green and was additionally enhanced by population transfer [11] with the same pulse as the CIDME pump pulse preceding the CIDME pulse sequence. Interestingly, the differences in the distance distributions between these two approaches are very small. In particular, the relative intensities of the peaks due to the second harmonic at $2.7 \mathrm{~nm}$ are comparable. There is only a small difference in the peaks due to the third harmonic at $2.35 \mathrm{~nm}$.

Altogether, the data shown in Fig. 5 approve that fairly large modulation depths are possible with the CIDME experiment. The largest modulation depth achieved with CIDME is actually due to the ability to observe and pump the central peak of the Gd spectrum at the same time. Such a setting is not possible in DEER, as a pump pulse resonant with the observed $A$ spins would change the refocusing position of the DEER echo. In the CIDME sequence, a pump pulse that is resonant with the $A$ spins does only invert the phase of the relevant virtual echo, but does not have an effect on the refocusing position of the virtual echo. This may lead to the hypothesis that a coupled spin pair that lies completely within the observation bandwidth takes the role of both pumped and observed spins: The first spin changes its resonance frequency by the dipolar coupling due to inversion of the second spin, whereas the second spin changes its frequency due to inversion of the first spin. However, such spin pairs will most likely not contribute to the CIDME modulation, because for spin pairs lying completely within the observation bandwidth, the observer $\pi$ pulses do not refocus the dipolar coupling $[35,36]$.

In any case, it is clear that a pump pulse with spectral overcears to a smaller mean separation between pump and observer frequencies. As a consequence, spin pairs which are not in weak coupling conditions contribute and introduce unwanted distortions to the dipolar spectrum [37-39]. Notably, the data obtained with the centered pump pulse (blue) showed the least number of oscillations in the time domain and the broadest peak in distance domain in comparison to the other data in Fig. 5. Further experiments towards this direction will be presented below in Section 3.1.4.

For the second important aspect, namely the presence of dipoarmonics, the presented CIDME data exhibit less harmonic content than RIDME experiments performed with the same Gdruler [31]. For CIDME with Gd-centers having smaller ZFS, excitation of higher harmonics may become more pronounced and even controllable, because the pump pulse covers a larger
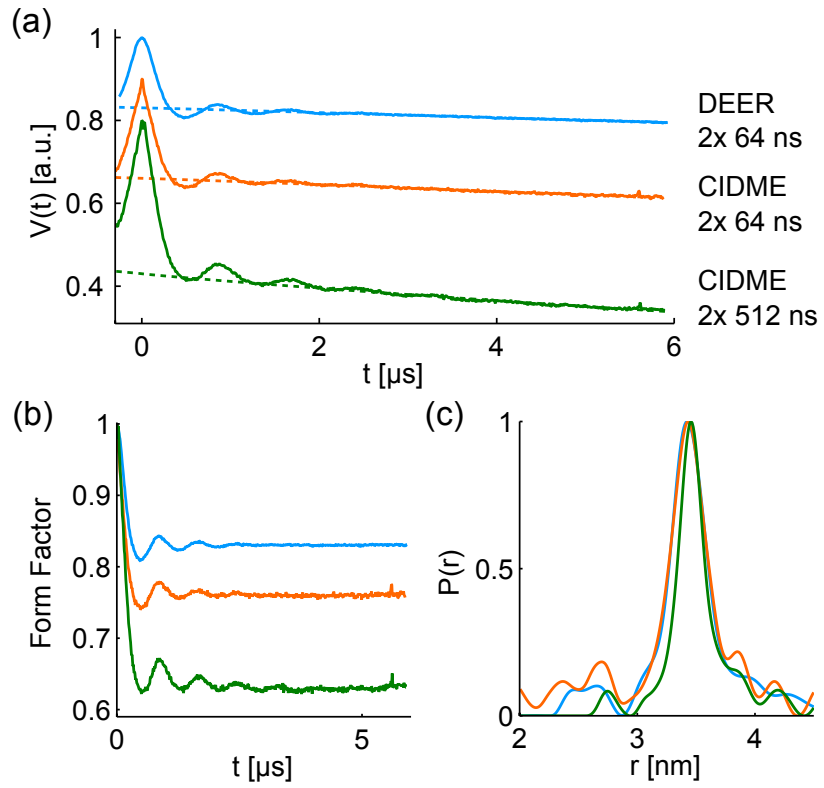

Figure 6: Comparison between DEER data and CIDME data of Gd-ruler $\mathbf{1}_{3}$ using consecutive pump pulses with $t_{\mathrm{p}}=64 \mathrm{~ns}, \Delta f=500 \mathrm{MHz}$ and an offset of $200 \mathrm{MHz}$ (blue and orange, respectively) as well as with $t_{\mathrm{p}}=512 \mathrm{~ns}, \Delta f=$ $1150 \mathrm{MHz}$ and an offset of $450 \mathrm{MHz}$ (green). (a) Primary data (solid lines), normalized and vertically offset for better visibility, together with background fits (dashed). The normalized echo amplitudes $V_{0}$ were $1.0,0.45$ and 0.35 for blue, orange and green, respectively. (b) Background-corrected form factors. (c) Distance distributions obtained with a regularization parameter of 1 .

higher harmonics while suppressing others. Such experiments would be of interest, since suppression of the fundamental frequency would be advantageous to acquire longer distances. The primary purpose of Gd-Gd CIDME is therefore to provide form factors that contain mainly the fundamental frequency.

\subsubsection{Comparison with DEER}

With the longer pulses in CIDME and a less severe echo reduction, it is a tempting question whether CIDME can outperform DEER despite the loss of a factor of two in $V_{\text {ref }}$ due to the longitudinal storage. For this purpose, we optimized both CIDME and DEER using the two-point optimization of $\eta_{2 \mathrm{P}}$ as described in [10]. The main outcome from this optimization was that CIDME with two consecutive pump pulses achieved a larger sensitivity than CIDME with a single pump pulse. Note that in order to avoid complications due to narrow pump-observer separation, only pump pulses which have no considerable overlap with the observed A spins were considered.

For two consecutive pump pulses with 64 ns duration each, we found good performance for both DEER and CIDME with $\Delta f$ $=500 \mathrm{MHz}$ and an offset of $200 \mathrm{MHz}$ between pump and observer. The DEER and CIDME results are illustrated in blue and orange in Fig. 6, respectively. When going to longer pulse durations, an asymptotic improvement in CIDME performance was observed based on $\eta_{2 \mathrm{P}}$. Two consecutive $512 \mathrm{~ns}$ long pulses with $\Delta f=1150 \mathrm{MHz}$ and an offset of $450 \mathrm{MHz}$ between pump and observer have therefore been tested with CIDME. The data 
obtained with these pulses are shown in green in Fig. 6 .

comparing the DEER and CIDME data obtained with the same pump parameters (blue and orange), the modulation depth with CIDME was larger. There are several reasons for this. First, there is a contribution to the dipolar modulation of a few percent that is present even without a pump pulse, as shown in zero time $t=0$ is different for DEER than for CIDME, as seen in the vertically displaced $V(t)$ curves in Fig. 6a. The DEER signal has a smooth curvature around $t=0$, as one would expect for the dipolar signal, which is composed of cosine funcby the chirp, frequency components from some orientations of the molecule may be missing, which could in principle reduce the apparent modulation depth in DEER. For the experimental parameters chosen here, however, this is fairly unlikely. The 570 CIDME signal, on the contrary, features a visible artificial spike at $t=0$, which does enhance $V_{0}$ and results in a larger apparent modulation depth.

Nevertheless, the larger modulation depth in CIDME is compensated by the smaller echo intensity $V_{0}$, which can even be

575 recognized by comparing the noise level in the form factors in panel (b). In fact, the CIDME echo amplitude $V_{0}$ was $45 \%$ of the DEER echo amplitude. Importantly, the relevant distance peak at $3.4 \mathrm{~nm}$ extracted from the CIDME and DEER data is indistinguishable (blue and orange). ment in modulation depth was achieved. However, the apparent modulation depth strongly depended on the background correction of primary data. Here, the background was fitted starting from $t_{\mathrm{bg}}=912 \mathrm{~ns}$ by a stretched exponential that corresponds to a dimensionality of the background $d_{\mathrm{bg}}=3.3$. For such a choice, the background-corrected form factor in panel (b) has an apparent low-frequency curvature and a modulation depth of $36 \%$ is obtained. If the background model parameters $t_{\mathrm{bg}}$ and $d_{\mathrm{bg}}$ are optimized automatically for best fit in the Deer0.88 remove the curvature in the form factor (data not shown). For such a small $d_{\mathrm{bg}}$, the background exhibits a peak at $t=0$ which reduces the apparent modulation depth to $21 \%$. The real modulation depth may lie between these two values. As one would expect, a RIDME experiment with the same $T$ of 1.124 $\mu$ s showed a similar background decay than the one observed here in CIDME (data not shown).

Even if a modulation depth of $36 \%$ for the long-pulse CIDME data is assumed, DEER sensitivity remains superior. In particular, CIDME achieved here only $75 \%$ of the sensitivity of the DEER experiment. For a distance of $3.4 \mathrm{~nm}$, where a total pulse duration of $128 \mathrm{~ns}$ is admissible in DEER, CIDME can therefore not outperform DEER. For shorter distances, however, the pump pulse in DEER needs to be shorter, such that CIDME the optimum conditions in terms of resonator bandwidth and available power may be different for CIDME than for DEER. In particular, one would expect that on a spectrometer with reduced power and bandwidth, where short chirp pulses no longer ration in CIDME improves sensitivity by significant enhancement of the modulation depth. An in-principle demonstration of such a situation is provided in the SI, where nitroxide-nitroxide CIDME is performed at deliberately reduced pulse power.

Another situation, where the performance of short chirp pulses constrains the DEER modulation depth, is when a large frequency separation on the order of $1 \mathrm{GHz}$ is required between pump and observer. With the longer pulse durations admissible in CIDME, the performance of the pump pulse, and thus the modulation depth, can be enhanced significantly. A large frequency separation between pump and observer is, for instance, important for the study of orientation selection at higher fields when using other spin labels than two Gd-complexes.

For pairs of Gd-complexes, a large frequency separation between pump and observer avoids contributions from spin pairs that are not in a weak coupling condition [37-39]. Interestingly, the long-pulse CIDME data presented here had a narrower distance distribution than the data obtained with the shorter pulses, which can even be recognized by counting the oscillations of the form factor in time domain. A similar observation is made for the data in Fig. 5. Using the same Gd-ruler, a narrowing of the distance distribution has also been observed for RIDME [31] as well as for direct excitation of multi-quantum transitions by an intense pump pulse in DEER (see [10]). Since the observed effects for the Gd-ruler $\mathbf{1}_{\mathbf{3}}$ with a Gd-Gd distance of $3.4 \mathrm{~nm}$ may be considered as rather small compared to typical distribution widths encountered in biological samples, we present data obtained with Gd-ruler $\mathbf{1}_{\mathbf{1}}$ in the following, which holds the two Gd-complexes at a distance of $2.1 \mathrm{~nm}$.

\subsubsection{Application to short distances}

The superior performance of CIDME for determination of short distances is exemplified using a $50 \mu \mathrm{M}$ solution of $\mathrm{Gd}-$ ruler 1 1. DEER experiments with monochromatic pulses at $\mathrm{W}$ band and at $\mathrm{Q}$ band of this particular compound revealed distance distributions broadened by pseudo-secular contributions [38]. Moreover, using a different Gd-ruler with smaller zero field splitting, a dual-mode resonator allowed to suppress this broadening by application of monochromatic DEER pump pulses with frequency separation up to $1 \mathrm{GHz}$ [39]. Based on our observations made for Gd-ruler $\mathbf{1}_{3}$ with chirp pulses, we would also expect such broadening for a chirp pump pulse having a small frequency offset. Accordingly, consecutive pulses with $t_{\mathrm{p}}=128 \mathrm{~ns}, \Delta f=600 \mathrm{MHz}$ and an offset of $200 \mathrm{MHz}$ were examined. The CIDME results shown in gray in Fig. 7 indeed reveal a broad distance distribution. In particular, the time-domain data in panel (a) shows less oscillations than the data obtained with Gd-ruler $\mathbf{1}_{\mathbf{3}}$.

To suppress this broadening, the pumping position was moved further away with consecutive pulses having $t_{\mathrm{p}}=512 \mathrm{~ns}, \Delta f$ $=500 \mathrm{MHz}$ and an offset of $1 \mathrm{GHz}$. At this larger offset, the results shown in black in Fig. 7 display a much narrower distance distribution. Broadening mechanisms due to spin pairs that are not in weak coupling conditions can therefore be suppressed substantially, which is in line with the experiments reported in [38, 39, 43]. It is the unique advantage of CIDME that a modulation depth larger than $10 \%$ can be achieved at 

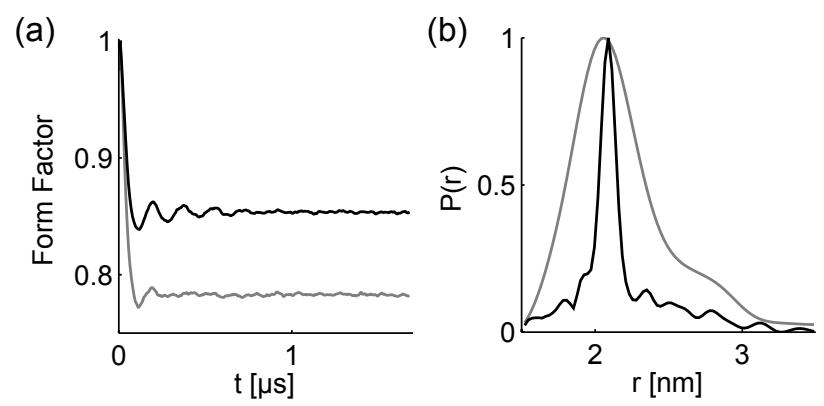

Figure 7: CIDME applied to Gd-ruler $\mathbf{1}_{\mathbf{1}}$ with an interspin distance of $2.1 \mathrm{~nm}$. Consecutive pulses with parameters $t_{\mathrm{p}}=512 \mathrm{~ns}, \Delta f=500 \mathrm{MHz}$ and an offset of $1 \mathrm{GHz}$ (black) as well as $t_{\mathrm{p}}=128 \mathrm{~ns}, \Delta f=600 \mathrm{MHz}$ and an offset of 200 $\mathrm{MHz}$ (gray) were examined. The observer pulses had durations of $8 \mathrm{~ns}$ and 16 ns. Despite the short evolution window, the delay $\tau_{2}$ needed to be set to $4.5 \mu \mathrm{s}$ due to a significant increase in a coherent artifact at $t=0$ at shorter $\tau_{2}$ (see SI). (a) Background corrected form factor. (b) Regularized distance distributions obtained with regularization parameters of 0.01 (black) and 10 (gray) based on the L-curve criterion $[41,42]$.

large offsets of $1 \mathrm{GHz}$, while observing the signal at the central peak of the Gd spectrum. For DEER with chirp pump pulses, one would expect much smaller modulation depths due to the restriction on pulse duration. In comparison to narrowing of the distance distribution by changing from DEER to RIDME $[31,43]$, it is essential that the principal dipolar modulation in CIDME is due to the fundamental frequency, whereas RIDME introduces dipolar harmonics that complicate data analysis. on RIDME at short $T$. Such short $T$ make the experiment prone to artifacts from additional coherence transfer pathways. In the example shown here, such an artifact at $t=0$ was suppressed by prolongation of the pulse delay $\tau_{2}$ beyond $\tau_{2}=2 \mu \mathrm{s}$, which is the delay that would suffice for the recorded evolution window. Further information and a preliminary explanation of this particular artifact is found in the SI.

\subsection{Nitroxide-nitroxide CIDME}

CIDME was also applied to nitroxide-nitroxide spin pairs compared to optimized DEER schemes with rectangular and chirp pump pulses. The experimental conditions when changing from $\mathrm{Gd}-\mathrm{Gd}$ experiments to nitroxide-nitroxide experiments change as follows: First, longitudinal relaxation of nitroxides at $50 \mathrm{~K}$ is slower than for $\mathrm{Gd}$ at $10 \mathrm{~K}$, so that one would not expect any RIDME contributions to the CIDME form factor for nitroxides at short delays $T$. As an example, nitroxide-nitroxide RIDME at low temperature was performed with much longer $T$ up to milliseconds [44] and relaxation agents were introduced to enhance longitudinal relaxation [45].

695 Second, a smaller excitation bandwidth is required with nitroxides due to the narrower spectra. However, the smaller transition moment of $S=1 / 2$ nitroxides as compared to $S=7 / 2$ Gd centers requires more energy input to the spin system for achieving the same excitation bandwidth. Moreover, the sep700 aration between pump and observer frequency windows becomes narrower for nitroxides and requires extra consideration on potential artifacts [36].
The experiments were performed with a WALP23 polypeptide inserted into DOPC bilayers with MTSL at residues 7 and 22. Since the lipids and buffers were not deuterated in this sample, CIDME was recorded without nuclear modulation averaging.

The optimized DEER scheme with a rectangular pump pulse was implemented according to [26]. In particular, the microwave resonator was adjusted for $Q_{\mathrm{L}}=300$ at $f_{0}=34.3 \mathrm{GHz}$ and pump and observation pulses with 12 ns duration and 100 $\mathrm{MHz}$ separation were placed symmetrically about the resonator center frequency. The placement with respect to the nitroxide spectrum is indicated in Fig. 8a.

For DEER with a chirp pump pulse and CIDME, a different scheme was used. In particular, the observation position was set to the central frequency of the resonator and to the maximum of the nitroxide spectrum for best detection sensitivity. The DEER pump pulse was a non-compensated $\operatorname{HS}\{6,1\}$ pump pulse with $t_{\mathrm{p}}=64 \mathrm{~ns}$, whereas the CIDME pump pulse had an extended duration of $t_{\mathrm{p}}=500 \mathrm{~ns}$. Based on $\eta_{2 \mathrm{P}}$ optimization [10], pulse parameters $\Delta f=200 \mathrm{MHz}$ and $150 \mathrm{MHz}$ were chosen for CIDME and DEER, respectively, both at an offset of 70 $\mathrm{MHz}$ (see also Fig. 8a).

Normalized primary data are shown in Fig. 8b, with DEER results using a monochromatic (blue) and a chirp (orange) pump pulse as well as CIDME data (green). While the DEER data are similar in shape, CIDME data reveal a curved background and a pronounced contribution at $t=\tau_{2}-\tau_{1}$. These features were also observed when recording CIDME without a pump pulse (gray), which corresponds to RIDME with $T=1 \mu \mathrm{s}$.

Background-corrected form factors are shown in panel $\mathrm{c}$, where CIDME data was cut off to exclude the artifact at $t=\tau_{2}-\tau_{1}$. When comparing the DEER datasets, modulation depth was highest with a monochromatic pump pulse. The larger modulation depth with the monochromatic pump pulse as compared to the chirp pump pulse is explained by the placement of pump and observation frequencies (compare Fig. 8a). The actual advantage of using the chirp pump pulse in the proposed DEER scheme becomes clear when comparing the SNR given by $\lambda / \sigma$, where $\sigma$ is the standard deviation of the form factor obtained from the fitting residual filtered by a $10 \mathrm{MHz}$ high-pass filter. In particular, the SNR with the chirp pump pulse was $28 \%$ higher than with the monochromatic pump pulse. This scheme is therefore very promising for the application of chirp pump pulses to nitroxide-nitroxide DEER at $\mathrm{Q}$ band. Important restrictions to this approach are (i) potential interference at short distances and (ii) spectral overlap between pump and observation frequencies, resulting in artifacts at $t=\tau_{2}-\tau_{1}$ (see DEER form factors in panel c).

With respect to interference, the utilized chirp pump pulse with $t_{\mathrm{p}}=64 \mathrm{~ns}$ reproduced the DEER distance distribution obtained with the monochromatic pulse rather well (see Fig. 8d). With respect to the artifacts at $t=\tau_{2}-\tau_{1}$, it is presumed that these can be suppressed further by also shaping the observer pulses. The situation is different for CIDME. As a first aspect, the pronounced artifact at $t=\tau_{2}-\tau_{1}$ and the curvature in the CIDME background inhibit a reliable data analysis. For instance, the background shape results in the artificial peak around $4 \mathrm{~nm}$ in the CIDME distance distribution in Fig. 8d. Both the back- 
(a)
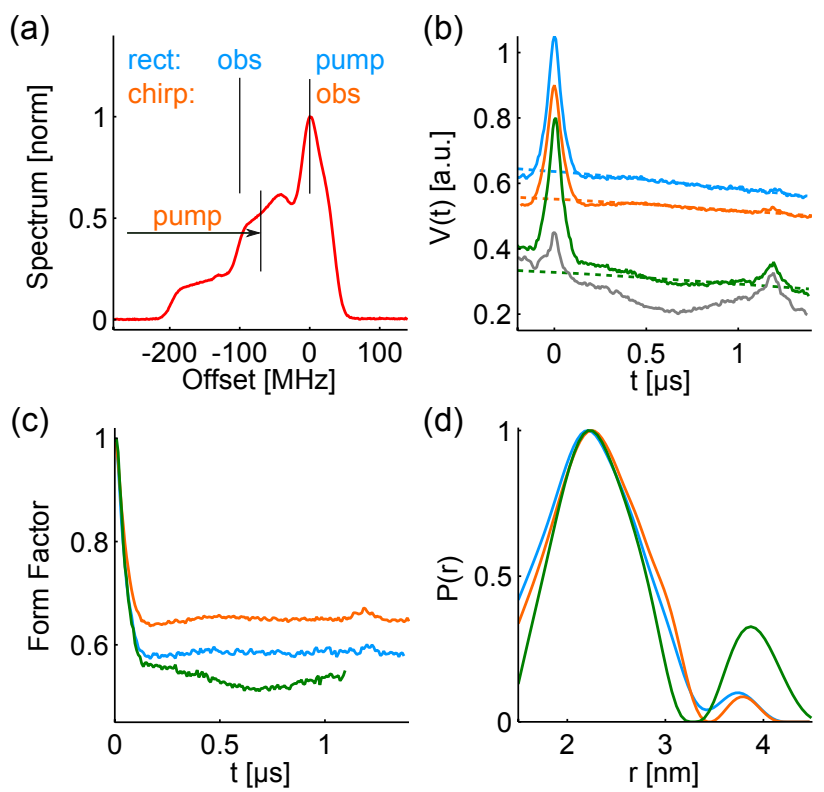

(d)

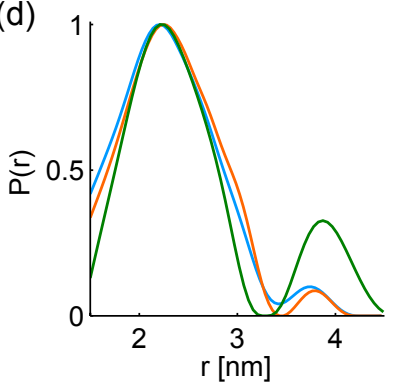

Figure 8: CIDME applied to WALP23 with MTSL at residues 7 and 22. (a) Field-swept echo-detected spectrum cast to a frequency axis with pump and observer positions used in DEER with either monochromatic or chirp pump pulses and CIDME. The chirp pump pulse used here was a non-compensated $\mathrm{HS}\{6,1\}$ pulse, with $t_{\mathrm{p}}=64 \mathrm{~ns}$ and $\Delta f=150 \mathrm{MHz}$ for DEER and $t_{\mathrm{p}}=512$ ns and $\Delta f=200 \mathrm{MHz}$ for CIDME. (b) Normalized primary data for DEER with monochromatic pump pulse (blue), DEER with chirp pump pulse (orange), CIDME (green), and CIDME without pump pulse (gray), which is a RIDME sequence at short $T=1 \mu \mathrm{s}$. (c) Background-corrected form factor according to the color coding used in panel $b$. CIDME data were cut off to exclude the $t=\tau_{2}-\tau_{1}$ artifact. (d) Distance distributions obtained with a regularization parameter of 100 .

ground shape and the artifact at $t=\tau_{2}-\tau_{1}$ were also visible in the RIDME reference measurement. Due to the long $T_{1}$ time, the residual dipolar modulation in this RIDME reference cannot be due to relaxation and must be of different origin. We observed that the residual modulation that refocuses at $t=0 \mathrm{~ns}$ can be reduced by prolonging the duration of the $\pi / 2$ pulses at (3) and (4) to project and release coherence (see SI). The modulation at $t=0 \mathrm{~ns}$ does therefore originate from inversion of unobserved spins by the $\pi / 2$ pulses in the $\pi / 2-T-\pi / 2$ block (see also [18, 33, 20]).

For the residual modulation that refocuses at $t=\tau_{2}-\tau_{1}$, we found that it depends both on $T$ and on the observation position (see SI). In particular, this modulation was most significant for small $T<10 \mu$ s at the central peak of the nitroxide spectrum and virtually absent for $T>20 \mu \mathrm{s}$. Similarly, the unexpected curvature of the background was only significant at very short $T$ $<2 \mu$ s. These observations corroborate the absence of such artifacts in nitroxide-nitroxide RIDME at longer $T$ [21, 18, 44, 46]. Clearly, one could therefore diminish the unwanted CIDME artifacts towards $t=\tau_{2}-\tau_{1}$ by (i) prolongation of $T$ or by (ii) observation at a different field position. However, both strategies entail an additional signal loss in the case studied here due to (i) pronounced background decay at longer $T$ mediated by matrix protons and (ii) reduced echo intensity at observation positions other than the maximum of the nitroxide spectrum.
Even if the artifacts could be reduced by prolongation of $T$, our results indicate that the sensitivity of nitroxide-nitroxide CIDME is unlikely to reach beyond the sensitivity of established Q-band nitroxide-nitroxide DEER with monochromatic pulses. In fact, the SNR achieved with CIDME was below the SNR achieved with DEER using a monochromatic pump pulse. Best sensitivity was achieved by the inverted DEER scheme employed here with a short HS $\{1,6\}$ pump pulse.

However, if experiments are performed on a Q-band spectrometer with reduced power and bandwidth, such that the optimized DEER schemes are not feasible, CIDME could become interesting in terms of sensitivity. The principle of sensitivity enhancement for nitroxide-nitroxide systems by CIDME under these conditions is demonstrated in the SI, where the experiments with the WALP sample have been performed at reduced pulse power.

\section{Conclusions and outlook}

CIDME has proven to be a technique that is free of interference effects related to the frequency-swept excitation of chirp pump pulses. This allows for improving the inversion efficiency of the pump pulse by prolongation of the pulse duration. However, bypassing interference by longitudinal storage comes at the price of loosing half of the echo signal. Accordingly, CIDME can only outperform DEER in terms of sensitivity, if the prolongation of the pump pulse can compensate for providing only half of the echo signal. CIDME must therefore achieve at least twice the modulation depth than DEER.

As demonstrated in our experiments using high-power Q-band instrumentation, short pump pulses with duration of $64 \mathrm{~ns}$ provided DEER sensitivity that was beyond CIDME sensitivity with longer pump pulses for both Gd-Gd and nitroxidenitroxide spin pairs. In particular, the optimized DEER schemes provided decent modulation depth, so that there was not much room for improvement when changing to CIDME. There are, however, a number of situations where such DEER performance is not attainable:

First, the experiment may require a large frequency separation between the pumped and observed spins. Accordingly, the pump pulse needs to be applied at the far shoulders of the resonator profile, where pulse performance becomes deteriorated. Examples are the study of orientation selection involving broad spectra, spectroscopically orthogonal spin labels, or the suppression of spin pairs that are not in weak coupling conditions. For the latter example, a Gd-Gd CIDME modulation depth larger than $10 \%$ was achieved at a frequency separation between pump and observer of $1 \mathrm{GHz}$.

Second, pulse excitation power may be reduced due to availability of high-power instrumentation, which is a particular challenge at higher fields [47, 48]. Accordingly, we expect CIDME to be a sensitive technique at higher fields. In particular, the study of orientation selection involving nitroxide spin labels may be performed at higher sensitivity when changing from DEER to CIDME. In addition, favorable CIDME sensitivity is expected for a number of transition metal ions with spin $S=1 / 2$, where even high-power instrumentation cannot result 
${ }_{840}$ in DEER modulation depths larger than $10 \%$ due to the broad spectra.

Besides sensitivity considerations, however, the applicability of CIDME is currently largely restricted by the artifacts observed at short storage times $T$. While these are at present not fully understood, it is important to note the similarity of the CIDME pulse sequence to the RIDME pulse sequence. We would therefore expect that a number of open questions on the origin of these artifacts become clarified by further investigations on spin dynamics of RIDME. One such example is the recent analysis of nuclear modulations in RIDME [32].

\section{Acknowledgments}

For the experimental part of this work performed at ETH Zurich, funding from the SNSF grant 20020_157034 is acknowledged. For the synthesis of Gd-rulers $\mathbf{1}_{\mathbf{n}}$, funding from the DFG priority programme SPP 1601 (grant GO 555/6-1) is acknowledged. Maxim Yulikov, Katharina Keller and Irina Ritsch are acknowledged for fruitful discussions on RIDME. Moreover, we are thankful to Luca Garbuio, Yevhen Polyhach, and Takuya Segawa for preparation of the spin labeled WALP le. Andrin Doll acknowledges a mobility grant from the SNSF for research abroad.

\section{References}

[1] M. Pannier, S. Veit, A. Godt, G. Jeschke, H. Spiess, Dead-time free measurement of dipole-dipole interactions between electron spins, J. Magn. Reson. 142 (2000) $331-340$.

[2] G. Jeschke, Y. Polyhach, Distance measurements on spin-labelled biomacromolecules by pulsed electron paramagnetic resonance, Phys. Chem. Chem. Phys. 9 (2007) 1895-1910.

[3] O. Schiemann, T. F. Prisner, Long-range distance determinations in biomacromolecules by EPR spectroscopy, Q. Rev. Biophys. 40 (2007) $1-53$

[4] G. Jeschke, DEER distance measurements on proteins, Annu. Rev. Phys. Chem. 63 (2012) 419-446.

[5] P. P. Borbat, J. H. Freed, Pulse dipolar electron spin resonance: Distance measurements, in: C. R. Timmel, J. R. Harmer (Eds.), Structure and Bonding, Vol. 152, Springer Berlin Heidelberg, 2013, pp. 1-82.

[6] J. Baum, R. Tycko, A. Pines, Broadband and adiabatic inversion of a twolevel system by phase-modulated pulses, Phys. Rev. A 32 (1985) 34353447

880 [7] M. Garwood, L. DelaBarre, The return of the frequency sweep: Designing adiabatic pulses for contemporary NMR, J. Magn. Reson. 153 (2001) 155 $-177$.

[8] A. Doll, S. Pribitzer, R. Tschaggelar, G. Jeschke, Adiabatic and fast passage ultra-wideband inversion in pulsed EPR, J. Magn. Reson. 230 (2013) $27-39$.

[9] P. E. Spindler, S. J. Glaser, T. E. Skinner, T. F. Prisner, Broadband inversion PELDOR spectroscopy with partially adiabatic shaped pulses, Angew. Chem. Int. Ed. 52 (2013) 3425-3429.

[10] A. Doll, M. Qi, N. Wili, S. Pribitzer, A. Godt, G. Jeschke, Gd(III)-Gd(III) distance measurements with chirp pump pulses, J. Magn. Reson. 259 (2015) $153-162$

[11] A. Doll, M. Qi, S. Pribitzer, N. Wili, M. Yulikov, A. Godt, G. Jeschke, Sensitivity enhancement by population transfer in Gd(III) spin labels, Phys. Chem. Chem. Phys. 17 (2015) 7334-7344.

[12] P. E. Spindler, I. Waclawska, B. Endeward, J. Plackmeyer, C. Ziegler, T. F. Prisner, Carr-purcell pulsed electron double resonance with shaped inversion pulses, J. Phys. Chem. Lett. 6 (2015) 4331-4335.

[13] P. P. Borbat, E. R. Georgieva, J. H. Freed, Improved sensitivity for long-distance measurements in biomolecules: Five-pulse double electronelectron resonance, J. Phys. Chem. Lett. 4 (2013) 170-175.
14] A. Doll, G. Jeschke, Fourier-transform electron spin resonance with bandwidth-compensated chirp pulses, J. Magn. Reson. 246 (2014) 18 26.

[15] A. Doll, G. Jeschke, EPR-correlated dipolar spectroscopy by Q-band chirp SIFTER, Phys. Chem. Chem. Phys. 18 (2016) 23111-23120.

[16] I. Tkach, G. Sicoli, C. Höbartner, M. Bennati, A dual-mode microwave resonator for double electron-electron spin resonance spectroscopy at Wband microwave frequencies, J. Magn. Reson. 209 (2011) 341 - 346.

[17] G. Jeschke, S. Pribitzer, A. Doll, Coherence transfer by passage pulses in electron paramagnetic resonance spectroscopy, J. Phys. Chem. B 119 (2015) 13570-13582.

[18] L. Kulik, S. Dzuba, I. Grigoryev, Y. Tsvetkov, Electron dipole-dipole interaction in ESEEM of nitroxide biradicals, Chem. Phys. Lett. 343 (2001) $315-324$

[19] W. Mims, Pulsed ENDOR experiments, Proc. R. Soc. A 283 (1965) 452457

[20] L. Kulik, Y. A. Grishin, S. Dzuba, I. Grigoryev, S. Klyatskaya, S. Vasilevsky, Y. D. Tsvetkov, Electron dipole-dipole ESEEM in fieldstep ELDOR of nitroxide biradicals, J. Magn. Reson. 157 (2002) 61-68.

[21] S. Milikisyants, F. Scarpelli, M. G. Finiguerra, M. Ubbink, M. Huber, A pulsed EPR method to determine distances between paramagnetic centers with strong spectral anisotropy and radicals: The dead-time free RIDME sequence, J. Magn. Reson. 201 (2009) 48 - 56

[22] A. V. Astashkin, Mapping the structure of metalloproteins with RIDME in: P. Z. Qin, K. Warncke (Eds.), Electron Paramagnetic Resonance Investigations of Biological Systems by Using Spin Labels, Spin Probes, and Intrinsic Metal Ions, Part A, Vol. 563 of Methods in Enzymology, Academic Press, 2015, pp. $251-284$.

[23] M. Qi, M. Hülsmann, A. Godt, Spacers for geometrically well-defined water-soluble molecular rulers and their application, J. Org. Chem. 81 (2016) 2549-2571.

[24] P. Lüders, H. Jäger, M. A. Hemminga, G. Jeschke, M. Yulikov, Distance measurements on orthogonally spin-labeled membrane spanning walp23 polypeptides, J. Phys. Chem. B 117 (7) (2013) 2061-2068.

[25] T. F. Segawa, M. Doppelbauer, L. Garbuio, A. Doll, Y. O. Polyhach, G. Jeschke, Water accessibility in a membrane-inserting peptide comparing Overhauser DNP and pulse EPR methods, J. Chem. Phys. 144 (2016) 194201

[26] Y. Polyhach, E. Bordignon, R. Tschaggelar, S. Gandra, A. Godt, G. Jeschke, High sensitivity and versatility of the DEER experiment on nitroxide radical pairs at Q-band frequencies, Phys. Chem. Chem. Phys. 14 (2012) 10762-10773.

[27] J. M. Böhlen, G. Bodenhausen, Experimental aspects of chirp NMR spectroscopy, J. Magn. Reson. 102 (1993) 293 - 301

[28] A. Raitsimring, A. Astashkin, J. Enemark, I. Kaminker, D. Goldfarb, E. Walter, Y. Song, T. Meade, Optimization of pulsed-DEER measurements for Gd-based labels: Choice of operational frequencies, pulse durations and positions, and temperature, Appl. Magn. Reson. 44 (2013) 649-670.

[29] A. Tannús, M. Garwood, Improved performance of frequency-swept pulses using offset-independent adiabaticity, J. Magn. Reson. A 120 (1996) 133 - 137

[30] M. Silver, R. Joseph, D. Hoult, Highly selective $\pi / 2$ and $\pi$ pulse generation, J. Magn. Reson. 59 (1984) 347 - 351

[31] S. Razzaghi, M. Qi, A. I. Nalepa, A. Godt, G. Jeschke, A. Savitsky, M. Yulikov, RIDME spectroscopy with Gd(III) centers, J. Phys. Chem. Lett. 5 (2014) 3970-3975.

[32] K. Keller, A. Doll, M. Qi, A. Godt, G. Jeschke, M. Yulikov, Averaging of nuclear modulation artifacts in RIDME experiments (submitted), J. Magn. Reson.

[33] K. Salikhov, S.-A. Dzuba, A. M. Raitsimring, The theory of electron spinecho signal decay resulting from dipole-dipole interactions between paramagnetic centers in solids, J. Magn. Reson. 42 (1981) 255-276.

[34] M. Yulikov, P. Lüders, M. Farooq Warsi, V. Chechik, G. Jeschke, Distance measurements in Au nanoparticles functionalized with nitroxide radicals and Gd3+-DTPA chelate complexes, Phys. Chem. Chem. Phys. 14 (2012) 10732-10746.

[35] A. Schweiger, G. Jeschke, Principles of Pulse Electron Paramagnetic Resonance, Oxford University Press, USA, 2001.

[36] K. Salikhov, I. Khairuzhdinov, Four-pulse ELDOR theory of the spin 1/2 label pairs extended to overlapping EPR spectra and to overlapping pump 
and observer excitation bands, Appl. Magn. Reson. 46 (2015) 67-83.

[37] A. Potapov, Y. Song, T. Meade, D. Goldfarb, A. Astashkin, A. Raitsimring, Distance measurements in model bis-Gd(III) complexes with flexible bridge. Emulation of biological molecules having flexible structure with Gd(III) labels attached, J. Magn. Reson. 205 (2010) 38 - 49.

[38] A. Dalaloyan, M. Qi, S. Ruthstein, S. Vega, A. Godt, A. Feintuch, D. Goldfarb, Gd(III)-Gd(III) EPR distance measurements - the range of accessible distances and the impact of the zero field splitting, Phys. Chem. Chem. Phys. 17 (2015) 18464.

[39] M. R. Cohen, V. Frydman, P. Milko, M. A. Iron, E. H. Abdelkader, M. D. Lee, J. D. Swarbrick, A. Raitsimring, G. Otting, B. Graham, A. Feintuch, D. Goldfarb, Overcoming artificial broadening in $\mathrm{Gd} 3+-\mathrm{Gd} 3+$ distance distributions arising from dipolar pseudo-secular terms in deer experiments, Phys. Chem. Chem. Phys. 18 (2016) 12847-12859.

[40] G. Jeschke, V. Chechik, P. Ionita, A. Godt, H. Zimmermann, J. Banham, C. Timmel, D. Hilger, H. Jung, DeerAnalysis 2006 - a comprehensive software package for analyzing pulsed ELDOR data, Appl. Magn. Reson. 30 (2006) 473-498.

990 [41] G. Jeschke, G. Panek, A. Godt, A. Bender, H. Paulsen, Data analysis procedures for pulse ELDOR measurements of broad distance distributions, Appl. Magn. Reson. 26 (2004) 223-244.

[42] Y.-W. Chiang, P. P. Borbat, J. H. Freed, The determination of pair distance distributions by pulsed ESR using tikhonov regularization, J. Magn. Reson. 172 (2005) $279-295$.

[43] A. Collauto, V. Frydman, M. D. Lee, E. H. Abdelkader, A. Feintuch, J. D. Swarbrick, B. Graham, G. Otting, D. Goldfarb, RIDME distance measurements using Gd(III) tags with a narrow central transition, Phys. Chem. Chem. Phys. 18 (2016) 19037-19049.

[44] L. Kulik, S. Paschenko, S. Dzuba, $130 \mathrm{GHz}$ ESEEM induced by electronelectron interaction in biradical, J. Magn. Reson. 159 (2002) 237 - 241.

[45] R. B. Zaripov, V. I. Dzhabarov, A. A. Knyazev, Y. G. Galyametdinov, L. V. Kulik, Use of additional fast-relaxing paramagnetic species for improvement of RIDME performance, Appl. Magn. Reson. 40 (2010) 11-19.

[46] A. Savitsky, A. A. Dubinskii, H. Zimmermann, W. Lubitz, K. Möbius, High-field dipolar electron paramagnetic resonance (EPR) spectroscopy of nitroxide biradicals for determining three-dimensional structures of biomacromolecules in disordered solids, J Phys. Chem. B 115 (2011) $11950-11963$.

[47] W. Hofbauer, K. A. Earle, C. R. Dunnam, J. K. Moscicki, J. H. Freed, High-power $95 \mathrm{GHz}$ pulsed electron spin resonance spectrometer, Rev. Sci. Instrum. 75 (2004) 1194-1208.

[48] P. A. S. Cruickshank, D. R. Bolton, D. A. Robertson, R. I. Hunter, R. J. Wylde, G. M. Smith, A kilowatt pulsed $94 \mathrm{GHz}$ electron paramagnetic resonance spectrometer with high concentration sensitivity, high instantaneous bandwidth, and low dead time, Rev. Sci. Instrum. 80 (2009) 103102 . 